\title{
Un acercamiento al estudio de las creencias de los profesores de lengua extranjera
}

\section{Mercedes Pizarro Carmona*}

Resumen: En los últimos 25 años, la didáctica de lenguas ha experimentado una importante evolución. Concretamente, el desarrollo de los estudios sobre la cognición ha permitido explorar las concepciones de los profesores acerca de la enseñanza y aprendizaje de una lengua extranjera (LE) y ha revelado que las creencias ejercen gran influencia en las decisiones que se toman en clase. Toda elección sobre qué y cómo enseñar, refleja las creencias del profesor sobre lo que piensa del proceso de enseñanza y aprendizaje de una lengua y ello influye en su actitud en el aula. Tomar conciencia sobre los pensamientos del profesor acerca de lo que cree eficaz en el proceso de enseñanza-aprendizaje de una lengua extranjera es un paso previo y fundamental para mejorar la labor docente.

Palabras clave: creencias, cognición, profesor, concepción, proceso de enseñanza-aprendizaje.

\begin{abstract}
In the last 25 years the field of language teaching has undergone a significant evolution. Specifically, the development of research on cognition has allowed exploring the teacher's beliefs on teaching and learning a foreign language and has revealed that their beliefs strongly influence their decisions. Teacher's choices about what and how to teach reflect their beliefs on teaching and learning a language; beliefs deeply impact the teacher's attitude in the classroom. Understand the teacher's own beliefs on what is efficient in teaching and learning a foreign language is a fundamental precondition to improve the teacher's work.
\end{abstract}

Key words: beliefs, cognition, teachers, conceptions, teaching-learning process.

* Licenciada en Lingüística Hispánica por la Universidad Complutense de Madrid España y Máster en Español Lengua Extranjera por la Universidad Antonio de Nebrija de Madrid, España. Actualmente trabaja en el Instituto Cervantes donde compagina la enseñanza de una lengua extranjera con la formación de profesores. 


\section{EL PROFESOR DE LENGUA EXTRANJERA Y SUS CREENCIAS}

A través de la evolución de la metodología y de la didáctica hemos visto que en el proceso de enseñanza-aprendizaje de una lengua extranjera no basta con priorizar un factor $u$ otro, sino que este proceso es un complejo entramado en el que intervienen muchos elementos a los que vienen a sumarse las ideas preconcebidas de los protagonistas del mismo. Como señala Marcelo (2000)

Los profesores tenemos una visión personal, un conjunto de valores y creencias sobre cómo se desarrolla el proceso de enseñanza y aprendizaje de la lengua y sobre cómo se apropian los estudiantes del conocimiento. Los profesores, al igual que otras personas orientan su conducta a partir del conocimiento y creencias que poseen y que se empieza a construir mucho antes de que decida dedicarse profesionalmente a la enseñanza.

Las creencias de los profesores por tanto, ejercen un papel importante en su práctica y es importante saber por qué actúa así, nos advierte Richards (2001)

The study of teacher's beliefs forms part of the process of understanding how teachers conceptualize their work. In order to understand how teachers approach their work it is necessary to understand the beliefs and principles they operate from.

Las aportaciones al panorama de la didáctica de lenguas extranjeras se han enriquecido con nuevas perspectivas y en los últimos años la investigación en didáctica también se ha abierto a nuevos ámbitos. Los estudios en didáctica se han preocupado por buscar explicaciones a muchos de los aspectos implicados en la enseñanza y el aprendizaje. Richards y Lockhart, (1994) señalaron precisamente que la exploración y el análisis de los procesos que acontecen en el aula, la investigación en acción, resulta fundamental para encontrar una explicación exacta de los mismos.

Este enfoque reflexivo se basa en la presuposición de que un profesor debe estar informado y poseer un conocimiento extenso de la enseñanza. Para lograr esto, la introspección puede ser de gran ayuda, ya que muchas veces los profesores desconocen en gran medida lo que ocurre en el proceso de enseñanza. A menudo no son conscientes del tipo de enseñanza que practican y para tener buena practica profesional no basta con la experiencia. Muchos profesores generan estrategias y rutinas sin que medie reflexión (Parker, 1984), sin reparar en que la reflexión crítica puede promover una comprensión más profunda de la docencia (Barlett, 1990; Wallace, 1991). Según esto, a través del análisis de las creencias y pensamientos que subyacen a las acciones de los profesores podremos llegar a comprender mejor su concepción de la enseñanza. Hay que tomar en cuenta que 
En lo que se refiere al pensamiento de los profesores acerca de la enseñanza y el aprendizaje, lo que se busca no es tanto perfilar la figura del profesor eficaz, sino explicar y comprender los procesos de enseñanza. (Halkes y Olson, 1984).

Como señalan los estudiosos del tema, en las últimas dos décadas, la investigación didáctica se ha centrado en el estudio de los procesos cognitivos y su influencia en la vida profesional de los docentes. Ello ha generado una importante línea de investigación. Borg (2003) recoge las cuestiones fundamentales hacia las que se dirigen las investigaciones sobre la cognición de los profesores. Entre ellas señala las siguientes: ¿En torno a qué giran los pensamientos del profesor y cómo se desarrollan?, ¿cómo influyen en su concepción de la enseñanza y del aprendizaje?, ¿cómo influyen en la práctica de clase, ¿qué entendemos por estudio de la cognición?

\section{EL CONCEPTO DE CREENCIA}

En el estudio sobre la cognición del profesor se han propuesto diversos términos para referirse a las ideas de los docentes, así como diversas definiciones sobre lo que significa el término creencia (percepciones, concepciones, ideaciones). En este trabajo se ha optado por el término creencia por considerarlo el más general, y en el que tienen cabida todos esos otros términos. Aquí se incluye una de las definiciones más completas y actuales sobre el concepto de creencia que es la que propone Ramos (2005):

Las creencias son ideas relativamente estables que tiene un individuo sobre un tema determinado, forjadas a través de su experiencia personal bajo la influencia de un proceso de construcción social, agrupadas en redes o sistemas, de cuya veracidad está convencido y que actúan como un filtro a través del cual percibe e interpreta el mundo que lo rodea, tomando sus decisiones de acuerdo con ello.

En el ámbito de la enseñanza y el aprendizaje de las lenguas tenemos creencias sobre cómo se aprende, qué utilidad tiene lo que se aprende, qué elementos son importantes en el proceso de aprendizaje. Los trabajos de Horwitz (1987) han resultado muy ilustrativos. El análisis de los datos que ha arrojado su cuestionario BALLI (Beliefs About Language Learning Inventory), aplicado en muchos otros trabajos, reflejan la idea de que en un sentido o en otro, profesores y alumnos tienen una idea preconcebida de lo qué es aprender y de lo qué es mejor hacer para lograrlo, llegando a conformar parte de la sabiduría popular. Ahora bien, todos los autores están de acuerdo en considerar que las creencias no se dan de forma aislada, sino que conforman sistemas de creencias relacionados entre sí. 


\section{EL SISTEMA DE CREENCIAS DE LOS PROFESORES}

Richards y Lockhart (1994) consideran que el sistema de creencias del profesor es la raíz de donde surgen gran parte de sus decisiones y actuaciones. Constituyen lo que se denomina "la cultura de la enseñanza", en donde están imbricados las creencias y los conocimientos relacionados con su trabajo.

Richards (1996) considera que existen dos tipos de conocimientos que influyen en cómo entienden la enseñanza y cómo la llevan a la práctica. Distingue entre dos tipos de conocimiento, uno relacionado propiamente con la asignatura, con el currículo y en cómo pueden o deben ser presentados los contenidos; el segundo tipo está relacionado con lo que el profesor considera que es una buena clase.

Cambrá (2003) habla de vida mental de los profesores y señala:

Les travaux développés depuis les années soixante-dix sur la vie mentale des professeurs (Calderhead, 1996; Clandinin \& Connelly, 1987; Clark \& Peterson,1986; Elbaz, 1985; Shavelson \& Stern, 1981) ont établi que les représentations permettent aux enseignants d'attribuer un sens à l'expérience, de guider les comportements et de gérer les dilemmes. Elles sont contextualisées, forgées à partir du vécu, chargées d'affectivité, anecdotiques_et même contradictoires_liées aux valeurs et ne coïncident pas toujours avec les savoirs scientifiques; elles ont un caractère personnel et spécifique mais aussi socioculturel et sont diffusées par les institutions et les médias.

Tomando esto en cuenta, cabe afirmar que los profesores están muy influidos por sus creencias (Williams y Burden, 1997) y que éstas están relacionadas con sus valores, su visión del mundo, y con el concepto que tienen de su posición en el mismo (Argyris y Schön, 1974). Los estudios existentes señalan, incluso, que estas creencias influyen más que los conocimientos que tienen los profesores en la manera en que planifican sus clases, en el tipo de decisiones que toman y en su labor general en el aula (Pajares, 1992). Aunque las creencias son reacias a cambiar, existen estudios que ponen de manifiesto el hecho de que el cambio en las creencias de los profesores precede a los cambios en la práctica (Richards, 2001).

La complejidad del estudio de las creencias también ha sido manifestada ya que se trata de un aspecto difícilmente observable en el aula. Algunos autores utilizan la metáfora del iceberg al hablar de este tema. A ello hay que añadir las dificultades asociadas al uso de medidas de evaluación de las creencias.

\section{ORIGEN DE LAS CREENCIAS}

La investigación sobre la cognición del profesor indica que más que un planteamiento metodológico determinado es su propia experiencia una de las razones que más influyen en su pensamiento y que condicionan su toma de decisiones. Resulta por 
tanto fundamental en el estudio de las creencias tener presente que se encuentra en la base de sus ideas. J. Richards y Lockhart y (1994) señalan:

La propia experiencia como estudiantes de lenguas.

La propia experiencia sobre qué planteamiento didáctico o enfoque metodológico funciona mejor.

La práctica establecida por los centros de trabajo o instituciones.

Factores de personalidad.

En esta misma línea, Johnson (1994) y Numrich (1996) señalan que muchas de las decisiones que toma el profesor sobre su actividad en el aula se basan en imágenes de profesores, materiales, actividades y organización de clase generadas por sus propias experiencias como estudiantes de una lengua extranjera.

Numrich (1996) en un estudio realizado con 26 profesores de inglés en prácticas sobre las percepciones de éstos acerca de sus necesidades durante la clase (Student teacher's percepcions of their needs during a practicum), señala que las decisiones del profesor sobre qué estrategias promover en clase se basan en la experiencia positiva o negativa que esas mismas estrategias le supusieron como estudiante. Como ejemplo señala que muchos de los profesores de su estudio consideraban importante integrar el componente cultural en sus clases, porque éste había sido agradable en su experiencia de aprendizaje. Al mismo tiempo, muchos de los profesores evitaban enseñar gramática o corregir errores porque sus propias experiencias en relación con esos aspectos habían sido negativas. Las investigaciones sobre la práctica en el aula sirven para reforzar y apoyar en buena medida lo anteriormente expresado.

Woods (1996) aporta su propia experiencia sobre lo que cree que funciona en el aula al hablar de sus creencias sobre la eficacia de los enfoques comunicativos y así lo recoge Borg (2003):

While years of formal instruction in French did not enable him to communicate in the language, six months in the company of French speakers developed his ability to do so.

También la formación que realizan los profesores puede hacerles descubrir un método y considerarlo eficaz e intentar incorporarlo a su práctica. Muchos autores plantean una cuestión que ha resultado central para el estudio sobre las creencias: investigar si la formación influye en las creencias y cómo éstas repercuten en la actuación en el aula. La cuestión resulta controvertida, existen estudios que aportan datos no determinantes recogidos en Borg (2003), Brookhart y Freeman (1992), Kagan (1992). En la mayoría de los casos, los investigadores han concluido que la formación pedagógica contribuye en las creencias de los jóvenes profesores, aunque la forma en que influye varía de unos a otros. 
A lo expuesto hasta aquí, habría que añadir el hecho de que la enseñanza es una actividad enmarcada en una serie de supuestos de índole cultural. El sistema cultural de un país influye en las actitudes del profesor hasta el punto de poder suscitarse un conflicto entre las diferentes creencias que tienen profesores y alumnos sobre el proceso de aprendizaje y enseñanza de una lengua.

Por otro lado, Borg (1998) plantea, en el estudio ya citado, cómo el entorno del centro de trabajo, las pautas del currículo, la política escolar, los planteamientos del lugar de trabajo y las disposiciones del centro hacen que el profesor tome decisiones sobre la planificación y el contenido, lo cual influye en su práctica docente y refleja sus creencias como profesor. Crookes y Arakaki (1999) señalan, por ejemplo, cómo las condiciones difíciles de trabajo afectan a lo que los profesores hacen. En un estudio con 20 profesores de inglés LE en un programa de inglés intensivo en Estados Unidos (The sources of ESL teacher's ideas for teaching) se analiza cómo un exceso de horas de trabajo por semana repercute en la dedicación a las horas de preparación. Ello ejerce una poderosa influencia a la hora de optar por una tendencia pedagógica.

Johnson (1996) en un estudio en Estados Unidos sobre las percepciones de los profesores en su primera etapa como docentes (A novice's perceptions of initial teaching experience) se refiere a cómo en ocasiones los profesores entran en conflicto con sus creencias al verse obligados a optar por una práctica pedagógica que no refleja en absoluto sus principios.

Por otra parte, Richards y Pennington's (1998) en un estudio realizado con cinco licenciados en inglés en Hong Kong analizan como los profesores de inglés de LE desarrollan sus actividades en su primer año de enseñanza (How ESL teachers coped with their first year of teaching). Muestran como el enfoque comunicativo, en el que fueron entrenados estos profesores, fue difícilmente aplicable en sus prácticas de clase debido a factores no tanto institucionales, como del propio entorno (estudiantes desmotivados, imposición del programa, presión de los exámenes, resistencia de los estudiantes a nuevas formas de aprender).

En cuanto a los factores de personalidad, algunos profesores prefieren ciertos modelos o estilos a la hora de enfocar las clases porque se ajustan más a su carácter. No obstante, el parámetro de la personalidad del profesor es uno de los que más controversia suscita. Algunos de los estudios que han investigado las creencias de los alumnos respecto a las cualidades de un buen docente, han coincidido en establecer que los estudiantes prefieren a los docentes que son amistosos, deseosos de ayudar y comunicativos, que al mismo tiempo, son ordenados, capaces de motivar y de mantener la disciplina (Weinstein, 1983).

Genovard y Gotzen (1990) establecen una serie de ideas positivas que resumirían la personalidad eficaz del profesor: dirige la acción, motiva, mantiene el interés, da retroalimentación inmediata, permite que el alumno aprenda a su 
propio ritmo, evita en lo posible la frustración y fracaso, promueve la transferencia del aprendizaje y desarrolla actitudes positivas en el alumno.

Aunque los estudios que intentan medir la eficacia docente relacionada con la personalidad parecen poco fiables, lo cierto es que los profesores también tienen su propia creencia sobre qué significa ser buen profesor o qué personalidades se ajustan mejor a cada tipo de grupo. Actualmente, los autores prefieren hablar de cuestiones relacionadas con estilos de enseñanza y expresividad del profesor. Las diferentes formas de desempeñar el papel docente representa una determinada posición que implica la adopción de una forma de enseñar. Así se suele hablar de estilos de enseñanza: autoritario/democrático; directo/indirecto; tradicional/progresivo; centrado en el profesor/centrado en el alumno.

La nueva perspectiva adoptada por los enfoques comunicativos requiere de profesores flexibles, dinámicos, activos, pedagógicamente formados, sin miedo a la innovación y a la investigación sobre su propia acción, que utilicen diferentes métodos y adapten su estilo de enseñanza al momento y contexto particular.

\section{TIPOS DE CREENCIAS}

Richards y Lockhart (1994) señalan que los estudios sobre las creencias de los profesores, generalmente, se han dirigido hacia los siguientes ámbitos: su percepción sobre los alumnos; sobre el aprendizaje y la enseñanza; sobre la lengua que enseñan; sobre el programa y el currículo; sobre sí mismos y sobre la enseñanza de lenguas como profesión.

Richards (2001) especifica y concreta las cuestiones hacia donde se dirigen las creencias respecto del aprendizaje y la enseñanza de una LE: enseñanza de la gramática, corrección de errores, destrezas, papel del profesor en la clase, práctica de clase. Como se observa en el cuadro 1. 
Cuadro 1. Áreas de estudio de las creencias

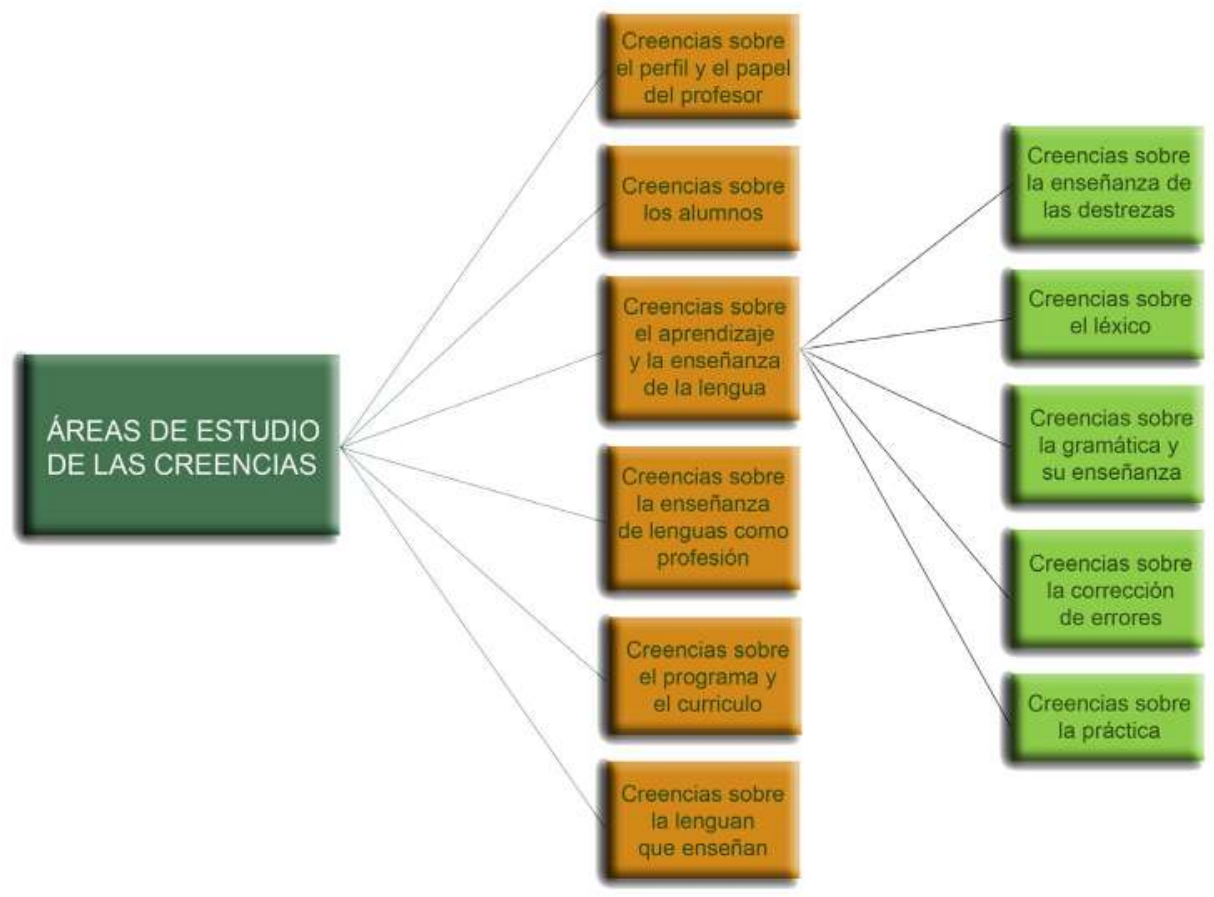

Creencias en torno al proceso de Aprendizaje de una lengua extranjera

Este tipo de creencias sobre el proceso de aprendizaje se relaciona directamente con lo que los docentes piensan y resulta eficaz para favorecer el aprendizaje e influye en su forma de enseñar.

Brindley (1984) señala que generalmente las concepciones de los profesores con respecto al aprendizaje suelen formarse a partir de su experiencia docente o también como señala Freeman (1992), desde de su experiencia como alumnos de lenguas. Como consecuencia de esa experiencia pasada podemos encontrarnos con profesores que optan por una forma de aprender en la que consideran fundamental el conocimiento explícito de la gramática, frente a otros que prefieren facilitar oportunidades para usar la lengua; con profesores que optan por un estilo o estrategia para sugerir a los alumnos, tal como deducir significados por el contexto, frente a memorizar vocabulario; con profesores que prefieran enseñar en pequeños grupos, frente a otros que consideren fundamental el trabajo individual; con profesores que optan por un papel específico para desempeñar en el aula como por ejemplo el papel de profesor como transmisor de conocimientos, frente al papel de facilitador del aprendizaje, entre otros. 
La propuesta de Williams y Burden (1997), basada en el constructivismo social, el cual tiene en cuenta la dimensión afectiva y cognitiva, considera que el aprendizaje eficaz se define de la siguiente manera:

Es un proceso complejo, produce algún tipo de cambio personal;

supone la creación de nuevas interpretaciones que son relevantes en el ámbito personal; puede asumir varias formas distintas; está siempre influido por el contexto en el que ocurre; es el resultado de la interacción social principalmente; a menudo es necesario que conlleve la mediación; difiere de un individuo a otro; es un proceso tanto emocional como cognitivo que dura toda la vida; está íntimamente relacionado con lo que las personas creen de sí mismas.

En una investigación realizada entre 106 profesores de lenguas inscritos en un programa de formación en una universidad británica (The implicit theories of experienced language teachers) Breen (1991) señala nueve principios que en opinión de los profesores facilitan el aprendizaje de la lengua:

1. prestar atención a la forma de la lengua (focus on form);

2. prestar atención al vocabulario o a los significados;

3. permitir que los estudiantes usen la lengua;

4. dirigir las capacidades de procesamiento mental de los estudiantes;

5. tener en cuenta el entorno afectivo del estudiante;

6. dirigir las necesidades e intereses de los estudiantes;

7. controlar el progreso de los estudiantes y ofrecerles retroalimentación;

8. promover la responsabilidad y la autonomía de los estudiantes;

9. sacar las lecciones y el grupo.

Richards (2001) describe las creencias de los profesores en torno al aprendizaje y la enseñanza de la lengua las cuales muestran una gran conciencia del papel central del estudiante en el contexto educativo. Incluso, cuando los profesores describen las características del buen profesor, lo hacen tomando como referencia las expectativas del alumno. Los encuestados ponen énfasis en señalar las relaciones entre alumno y profesor y la formación del profesor. Señalan como importante la participación del estudiante, el aprendizaje cooperativo.

En otra investigación realizada para explorar los procesos de cambio en las creencias de los profesores (Exploring teacher's beliefs and the process of change) mediante un cuestionario pasado a un grupo de 112 profesores de países del sureste asiático, este mismo autor, señala que los datos obtenidos revelan que entre las creencias de los profesores relacionadas con la enseñanza y el aprendizaje de la lengua, las más importantes para éstos son en este orden, las siguientes: 
1. La función de la gramática y la enseñanza de la gramática.

2. Las creencias sobre sus estudiantes.

3. Las destrezas de la lengua.

4. Las características del profesor.

5. El ambiente de clase / las condiciones de aprendizaje de la lengua.

6. Los objetivos de la enseñanza y el aprendizaje de la lengua.

7. La planificación / programación de la enseñanza.

8. Los métodos de enseñanza.

9. El papel de la práctica en el aprendizaje.

10. Otros: filosofía personal, errores de los estudiantes, la lengua en sí, procesos de enseñanza y aprendizaje

Es evidente que los resultados de su estudio no pueden generalizarse ni ser contrastados con la práctica de clase de esos profesores encuestados, pero pensamos que los datos que ofrecen dan puntos importantes y creemos que podrían ser una buena aproximación a la concepción de otros muchos docentes.

\section{CONCLUSIÓN}

Como decíamos al inicio de este trabajo, en los últimos años la investigación en didáctica ha revelado que las creencias ejercen gran influencia en las decisiones sobre la instrucción en clase y la práctica en el aula. Por eso, estamos convencidos de que un paso importante para acercarnos a la compresión del proceso de enseñanza-aprendizaje de una lengua extranjera consiste en preguntarnos cómo se apropian los conocimientos y cómo influimos los profesores en dicho aprendizaje. Por otro lado, tomar conciencia sobre las bases psicológicas que influyen y subyacen en la práctica de los profesores en el aula puede proporcionar un concepto más amplio de lo que significa enseñar y esto redundará en una mejora de nuestra labor docente. Muchas veces la inercia y la rutina no nos permiten ver nuevos ámbitos en los cuales intervenir; nuestras creencias, unas veces consciente y otras inconscientemente, dirigen nuestra labor, cerrándonos, muchas veces, a otras posibilidades. La reflexión y conciencia es un paso previo para el cambio en nuestra forma de pensar y posteriormente nuestra forma de actuar como docentes. 
BibLIOGRAFÍA

Aguirre Beltrán, B. (2004) Análisis de necesidades y diseño curricular. Vademécum para profesores de español como lengua segunda. Madrid: SGEL.

Borg S., (1998). Teacher's Pedagogical Systems and Grammar Teaching: A Qualitative Study. Tesol Quarterly, 32, (1).

, (1998) Talking about Grammar in the Foreing Language Classroom. Language Awareness, 7, (4).

(1999) Teacher's theories in grammar teaching. ELT Journal, 53, (3), Oxford University Press.

, M., (2001) Teacher's beliefs, en ELT Journal, 55, (2), Oxford University Press.

(2003) Teacher cognition in language teaching: A review of research on what language teachers think, know, believe, and do. [en línea] <http://www.personal. leeds.ac.uk/ edusbo/cognition/Articles/Language\%20Teaching\%2036- 2.pdf> , (2003) Teacher Cognition in Grammar Teaching: A Literature Review. [en línea] <http://www.personal.leeds.ac.uk/ edusbo/cognition/Articles/LA\%2012-2.pdf>

Ellis, R. (2008) La adquisición de segundas lenguas en un contexto de enseñanza. Análisis de las investigaciones existentes. III Coloquio internacional Programa EDICE. Valencia: Universidad de Valencia/Universidad Politécnica de Valencia.

Farrell, T.S.C. (2005) Conceptions of Grammar Teaching: A case study of Teacher's Beliefs and Classroom Practices. Teaching English as a second or foreing language. [en línea] <http://tesl-ej.org/ej34/a9.pdf>

García Santa-Cecilia, Á. (2002) Lengua y comunicación: tres décadas de cambio. El español en el mundo. Bases para una Europa plurilingüe. Anuario del Instituto Cervantes. Madrid: Consejo de Europa, Marco Común Europeo de Referencia para las lenguas: aprendizaje, enseñanza, evaluación.

Martín Peris, E. (s/f). El profesor de lenguas extranjeras: papel y funciones. [en línea] <http://www.upf.edu/pdi/dtf/ernesto.martin/archivos/articulos/ensenanza_alumno.pdf>

Pujols, M. y otros, (1998) Adquisición de lenguas extranjeras: perspectivas actuales en Europa. Edelsa.

Ramos Méndez, C. (2005) Ideaciones de estudiantes universitarios alemanes sobre su proceso de aprendizaje de español como lengua extranjera ante una enseñanza mediante tareas. Tesis. España: Universidad de Barcelona.

Richards J. (2001) Exploring teacher's beliefs and the processes of change. [en línea] <http://www.professorjackrichards.com/pdfs/exploring-teacher-change.pdf> 
(2003) 30 Years of TEFL/TESL: A personal reflection. [en línea]

<http://www.professorjackrichards.com/pdfs/30-years-of-TEFL.pdf>

Richards J. y Rodgers T. (1998) Enfoques y métodos en la enseñanza de idiomas. Londres: Cambridge University Press.

Richards J. y Lockhart J. (1994) Estrategias de reflexión sobre la enseñanza de idiomas. Londres: Cambridge University Press.

Sánchez Pérez, A. (2004) Enseñanza y aprendizaje en la clase de idiomas. SGEL.

Williams M., y Burden R.L. (1999) Psicología para profesores de idiomas. Enfoque del constructivismo social. Londres: Cambridge University Press. 